\title{
Accessibility aspects in UIDLs
}

\author{
David Faure • Paul Fogarassy-Neszly • \\ Costin Pribeanu $\cdot$ Jean Vanderdonckt
}

Published online: 28 June 2013

(c) Springer-Verlag Berlin Heidelberg 2013

A User Interface Description Language (UIDL) consists of a specification language that describes various aspects of a user interface under development [1]. Fundamentally, accessibility means that peoples with different disabilities can use a specific product. Particularly, in order to be accessible, a user interface must be perceivable, operable, and understandable for users, including those with disabilities or functional limitations as visual, auditory, physical, speech, cognitive, and neurological disabilities.

User interfaces accessibility can be approached through usability. International organization for standardization (ISO) 9241-11 defines usability as the extent to which a product can be used by the specified users to achieve specified goals effectively, efficiency, and with satisfaction in a specified context of use [2]. Accessibility focuses on including people with disabilities as the specified users and

\section{Faure}

Thales Research and Technology France, Campus de

Polytechnique, 1 rue Augustin Fresnel,

91767 Palaiseau cedex, France

e-mail: david.faure@thalesgroup.com

P. Fogarassy-Neszly

BAUM Engineering srl, Str. Traian Mosoiu 8,

310175 Arad, Romania

e-mail:pf@baum.ro

C. Pribeanu $(\bowtie)$

National Institute for Research and Development in Informatics-ICI Bucharest, Bd. Maresal Averescu 8-10, Bucharest, Romania

e-mail: pribeanu@ici.ro

\section{J. Vanderdonckt}

Louvain School of Management (LSM), Université catholique

de Louvain (UCL), Place des Doyens, 1,

1348 Louvain-la-Neuve, Belgium

e-mail: jean.vanderdonckt@uclouvain.be a wide range of situations, including assistive technologies, as the specified context of use.

In this special issue, the authors present several approaches regarding the model-based design of accessible user interface design and accessibility evaluation.

Van Hees and Engelen, in their paper entitled "Equivalent representations of multi-modal user interfaces-Runtime Reification of Abstract User Interface Descriptions", present a novel approach concerning the use of abstract user interface descriptions called Parallel User Interface Rendering (PUIR). The mechanism provided by PUIR is based on a single-consistent abstract model, offering a method to render the user interface simultaneously in multiple modalities. Each representation of the user interface provides equivalent semantics to ensure that collaboration between users of different modalities is facilitated. PUIR builds on research and development of abstract user interfaces and UIDLs, and it provides a framework where nonvisual rendering of the UI operates at the same level as the visual rendering rather than as a derivative. The current proof-of-concept implementation is based on a custom UIDL, but for further development collaboration with a UIDL is expected. The decision to continue with a broader interpretation of the original GUI design principles drives the choice of the underlying UIDL. The ability of, e.g., UsiXML to capture the UI with models at different levels of abstraction can be a real asset to this novel approach. The PUIR framework can contribute to the field of accessibility beyond the goal of providing visual impaired people access to GUIs. The generic approach behind the PUIR design lends itself well to developing alternative rendering agents in support of other disability groups.

The paper "Stories and signs in an e-learning environment for deaf people" by Bottoni et al. raises specific accessibility issues related to deaf peoples in the context of 
the development of a deaf-centered e-learning environment (DELE). One of the principles of DELE is to avoid text, whenever possible and not explicitly required by the learning activity, and to opt for a visual presentation of information. DELE design is based on conceptual metaphors, according to the embodied cognition paradigm, as well as on storytelling. Navigational, graphical, and activity structures in the story pages are specified via the UsiXML UIDL, which provides several models for the representation of the different aspects of DELE design, namely task coordination, flexible style of presentation, as well as typical e-learning. In addition, a meta-model for the domain specific language of stories is expressed in the form of a conceptual class diagram. The types in the meta-model are associated with specific patterns, relating the definition of stories as paths to the concrete organization of the interface. Authors propose a fully iconic page structure in order to enhance deaf people's motivation while navigating in virtual environments. The written representation of sign languages has been taken into account, proposing a meta-model for the sign-writing code extendible to sign languages in general. This can provide the basis for incorporating specifications for this form of interaction into UsiXML.

Calleros et al. in their paper entitled "Advancing Human Machine Interface Automatic Evaluation", develop a format of accessibility and usability guidelines that is common to all stakeholders, so as to facilitate the communication of guidelines and their evolution over time, and guarantee that a same guideline will be understood, interpreted, and evaluated in the same way. Their evaluation tool is supposed to addresses main shortcomings, such as the support of multiple bases of guidelines for accessibility and usability on-demand, with different levels of details. Calleros et al. propose an approach to automated UI evaluation with a cognitive architecture, usable for industrial applications. It uses a model-driven approach and is connectable with tools already in use, such as Matlab or Scade, allowing the re-use of the models defined by the system designers. The model of the user interface, as described in their article, can be further developed in order to test different interfaces configurations, and these models together can be used for automated user interfaces evaluation. Still an open issue is the connection between the cognitive architecture system (CASCaS) and UsiXML.

Kaklanis et al. introduce a framework for automatic simulated accessibility and ergonomy evaluation of virtual prototypes using virtual user models able to describe efficiently the interaction of virtual users with disabilities and the elderly with the virtual prototype within a virtual environment. A set of accessibility and ergonomy metrics are proposed. Experimental results on the accessibility and ergonomy evaluation of different workplace designs for the use of a telephone, and a stapler show how their proposed framework can be put into practice and demonstrate its significant potential. The proposed virtual user-modeling framework describes virtual humans focusing on the elderly and people with disabilities. The concepts of the Abstract User Model, Generic Virtual User Model, and instance of a Generic Virtual User Model, Primitive Task, Task Model, and Simulation Model are introduced, describing the characteristics of the virtual-disabled user, how a task of the user can be executed, and the simulation scenario to be followed during the simulation process, respectively. Experimental results showed how the framework can be put into practice and reveal its significant potential. The use of UsiXML for the implementation of all the models makes the entire framework very generic, as UsiXML can accurately describe the user interfaces to be evaluated, the user tasks, and the simulation scenario to be executed as well as the user itself in an abstract way, which does not contain any implementation details.

Miñón et al. describes a tool, called SPA4USXML (Service Provider Annotations for Ubiquitous Services through UsiXML), aimed at graphically creating instances of task models, abstract user interfaces, and multimedia resource models. The tool is supposed to be fed with descriptions of services provided by ubiquitous environments and web services. Its main goal is to assist service designers to create abstract specifications of the services for the Egoki adaptive system. SPA4USXML assists the service designer in the process of creating abstract user interfaces and enables the design process to take place in a faster and intuitive way, allowing the detection of errors at design time and facilitating the modification of the resulting interfaces. In addition, the tool allows the services to be enriched with additional resources that follow a model with an "Access for All" approach to ensure accessibility and adaptability. The case study described by Miñón et al. demonstrates that SPA4USXML reduces the necessary time to create task models, abstract user interface models, and resource models. Also, the tool avoids the need for the service designers to learn the UsiXML syntax and the XML technology in detail, since the tool's graphical interface abstracts the underlying syntax. Furthermore, their research demonstrates that accessibility requirements can be integrated in the design phase, both extending the meta-models and extracting semantic data in the transformation process.

\section{References}

1. Guerrero-Garcia, J.: A theoretical survey of user interface description languages: preliminary results, Web Congress. LAWEB '09. Latin American, pp. 36-43 (2009)

2. International Organization for Standardization ISO 9241-11. Ergonomic requirements for office work with visual display terminals, part 11: guidance on usability (1998) 